\title{
Avian Versus Mammalian Sleep: the Fruits of Comparing Apples and Oranges
}

\author{
Niels C. Rattenborg • Dolores Martinez-Gonzalez
}

Published online: 23 November 2014

(C) Springer International Publishing AG 2014

\begin{abstract}
Insight into the functions of sleep in humans can be gained through studying sleep in animals. In contrast to model-based approaches which emphasize similarities between sleep in humans and animals amenable to experimental manipulation, comparative-based approaches give equal emphasis to the similarities and differences in sleep across the animal kingdom, and thereby aim to reveal overarching principles not readily apparent using other approaches. Avian sleep serves as a prime example. Birds independently evolved rapid eye movement (REM) and non-REM sleep, states that are in many, but, importantly, not all respects comparable to those in mammals. When compared to the sleep traits specific to one group, those shared by mammals and birds are more likely to be involved in fundamental functions. In this review, we summarize recent advances in our understanding of avian sleep, and the implications that such comparative work has for understanding sleep in mammals, including ourselves.
\end{abstract}

Keywords Bird $\cdot$ Reptile $\cdot$ Mammal $\cdot$ Non-rapid eye movement $\cdot$ Rapid eye movement $\cdot$ Sleep .

Electroencephalogram $\cdot$ Slow oscillation $\cdot$ Rhythms ·

Traveling · Slow-wave activity · Deprivation · Homeostasis · Local $\cdot$ Evolution $\cdot$ Function $\cdot$ Memory $\cdot$ Hippocampus · Sleeplessness · Ontogeny · Development · Nidopallium · Prefrontal cortex $\cdot$ Flight $\cdot$ Model $\cdot$ Comparative

This article is part of the Topical Collection on Function of Sleep

N. C. Rattenborg $(\bowtie) \cdot$ D. Martinez-Gonzalez

Avian Sleep Group, Max Planck Institute for Ornithology,

Eberhard-Gwinner-Stasse 5, 82319 Seewiesen, Germany

e-mail: rattenborg@orn.mpg.de

D. Martinez-Gonzalez

e-mail: martinez@orn.mpg.de

\section{Introduction}

Our understanding of the human brain during health and illness will remain incomplete until we understand the purpose of sleep. Insight into the mechanisms and functions of sleep in humans can be gained through examining sleep across the animal kingdom. Research on sleep in animals employs either model-based or comparative-based approaches. Model-based approaches aim to gain insight into human sleep through examining human-like sleep states in animals amenable to experimental manipulation. Historically, this line of research has focused on mammals such as rodents. More recently, the fruit fly (Drosophila melanogaster), wherein the genetic dissection of sleep can be more readily performed, has become a powerful model for examining sleep $[1,2]$. The utility of model organisms, in general, is often viewed as constrained by the degree to which their sleep mimics that in humans. For example, although "simple" animal models, such as Drosophila, may provide insight into the initial, perhaps cellular functions of sleep, from a strictly model-based perspective, their contribution to our understanding of potential secondarily evolved functions of sleep and its sub-states, rapid eye movement (REM) and non-REM (NREM) sleep, present in humans and other mammals is often viewed as limited given the absence of comparable brain activity in sleeping Drosophila [3]. Nonetheless, even when model organisms (Drosophila, rodents, etc.) fail to mimic all aspects of human sleep, or discoveries made in model organisms fail to translate directly to humans, they retain comparative value that can inform our understanding of sleep in general, and thereby, sleep in ourselves.

Indeed, in contrast to model-based research that emphasizes similarities and, in our opinion, often shies away from potentially more informative differences, comparative-based research gives equal emphasis to similarities and differences across all taxonomic groups in an attempt to reveal overriding 
principles that might remain obscure using a more narrow model-based approach [4, 5]. For example, only birds exhibit two sleep states readily comparable to mammalian NREM and REM sleep, a similarity that evolved via convergent evolution [6]. Consequently, clues to the mechanisms and functions of these states can be gained through identifying other traits that evolved independently in mammals and birds [7]. Similarly, this comparative approach can also identify potential red herrings arising from a strictly mammalian-based approach that could draw attention away from more fundamental traits linked to sleep as it manifests in mammals and birds. For example, birds exhibit mammalian-like sleep-related EEG activity, despite lacking the laminar arrangement of neurons found in the neocortex [8•]. Consequently, birds tell us that the neocortex is not necessary to generate these rhythms, and therefore, they are unlikely to perform functions unique to this pattern of neuronal organization and associated computational properties. Even if functional theories based on traits found only in mammals are not chasing red herrings, their failure to explain aspects of sleep shared by mammals and birds, suggests that they may be missing more fundamental functional components of NREM and REM sleep [9•]. In these respects, the comparative research on avian sleep summarized herein, as well as comparative sleep research in general, can inform our understanding of sleep in mammals. Finally, comparative research has the potential to reveal unexpected discoveries, such as unihemispheric slow-wave sleep in dolphins [10] and adaptive sleeplessness in sandpipers [11••] that challenge our current thinking on sleep, and thereby provoke new lines of thought and experimentation that ultimately enhance our understanding of sleep and performance in ourselves $[12-15,16 \bullet, 17 \bullet]$.

\section{Similarities Between Avian and Mammalian Sleep}

\section{NREM Sleep}

The similarities between avian and mammalian sleep are many. During NREM sleep (also called slow-wave sleep in birds) the EEG shows high-amplitude slow waves similar to those observed in mammals. As in mammals [18], avian slow waves seem to reflect the slow oscillation of neuronal membrane potentials between depolarized "on-states" with action potentials, and hyperpolarized "off-states" with neuronal quiescence [19]. Also, like mammals, including humans [20], a recent study revealed that slow waves propagate as traveling waves through the avian brain, despite marked differences in neuronal cytoarchitecture between the two groups [8•]. In diurnal songbirds, EEG slow-wave activity (SWA) (spectral power between 0.5 and $4.5 \mathrm{~Hz}$ ) is highest at the beginning of the night and gradually declines with time spent in NREM sleep [21-23], suggesting mammalian-like homeostatic regulation. Nonetheless, until recently, it had been unclear whether avian SWA increases following sleep deprivation. In contrast to an earlier study that did not detect an increase in SWA following $24 \mathrm{~h}$ of sleep deprivation [24], an increase in SWA was recently shown in pigeons (Columba livia) following short-term $(8 \mathrm{~h}$ ) sleep deprivation [25], the first experimental evidence for the homeostatic regulation of SWA in birds. This difference may be due to the duration of sleep deprivation, as some mammals show an increase in SWA following short-term, but not long-term sleep deprivation [26]. Subsequently, a similar response to short-term sleep deprivation was observed in white-crowned sparrows (Zonotrichia leucophrys gambelii) [27], suggesting that as in mammals [28], this is a general feature of avian sleep [29]. Finally, as in humans and other mammals wherein SWA increases locally in the neocortex in response to localized stimulation [30], it was recently shown that the increase in SWA observed following sleep deprivation in birds also depends on the degree of stimulation during wakefulness. Pigeons kept awake for $8 \mathrm{~h}$ while watching David Attenborough's, The Life of Birds with only one eye, showed an increase in SWA during recovery NREM sleep only in the visual part of the brain (hyperpallium) receiving input from the stimulated eye $[31 \bullet \bullet]$. Collectively, these studies show that the local, use-dependent homeostatic regulation of SWA is a fundamental feature of NREM sleep.

\section{REM Sleep}

Avian and mammalian REM sleep also shares many similarities. REM sleep in both taxa is associated with low-amplitude, high-frequency EEG activity similar to that occurring during wakefulness [23-25, 27, 32•]. During such periods of EEG activation, birds show behavioral signs of reduced muscle tone, such as a gradual drop of the head [32•, 33, 34]. Like mammals, behavioral thermoregulatory responses such as shivering and panting, are suspended during REM sleep, perhaps as a result of reduced muscle tone [32•, 35, 36]. Finally, REM sleep is associated with rapid eye movements (except in species with limited eye movements, such as owls) and occasional twitches [32•].

The timing and amount of avian REM sleep appears to be under both circadian and homeostatic regulation. In primarily diurnal species, the time spent in REM sleep increases across the night [21-25]; although discrepancies in this pattern have been reported [37]. In at least some birds, the increase in REM sleep across the night reflects both an increase in the incidence and duration of REM sleep bouts [23, 25]. Although the forced desynchrony experiments needed to firmly establish the circadian regulation of REM sleep [38] have not been performed in birds, these findings at least suggest that the timing of REM sleep is regulated by the circadian rhythm in both mammals and birds. 
In addition to a possible circadian influence, avian REM sleep also appears to be under homeostatic control. Tobler and Borbély [24] were the first to show an increase in REM sleep following $24 \mathrm{~h}$ of deprivation in pigeons. REM sleep also increases following shorter periods of sleep deprivation in pigeons [25]. In addition, pigeons subject to chronic sleep restriction by the disk-over-water method [39], or a modified, more effective version [40], show large increases in REM sleep during recovery similar to those observed in rats sleep deprived using the same methods. Interestingly, in contrast to rats that develop a syndrome characterized by skin lesions, increased energy expenditure, and ultimately death [41], pigeons do not develop signs of this syndrome; although energy expenditure was not measured directly in the pigeon studies. Also, it remains unclear whether these findings reflect a true mammalian (rat)/avian (pigeon) difference, or differences in the degree to which pigeons and rats were exposed to the water during deprivation, a thermal challenge that might interact with sleep deprivation to produce aspects of the syndrome. Finally, it is possible that pigeons were able to partially circumvent the procedure by "leaking" SWA into wakefulness [42]. Although it remains unclear whether mammals and birds differ with regard to the sleep deprivation syndrome, these short- and long-term sleep deprivation studies nonetheless clearly show that REM sleep increases following sleep loss in both taxa.

The development of REM sleep is also similar in mammals and birds. In mammals, the amount of REM sleep is highest early in life and then declines to adult levels as the animal matures [43-46]. This occurs in utero and after birth in altricial species and primarily in utero in precocial species [47-51]. Although early studies reporting an age-related decline in REM sleep in birds were confounded by temperature [37] or other factors, such as the act of hatching that could influence the expression of REM sleep [32•], a recent EEGbased study of 66 barn owl (Tyto alba) nestlings in the wild revealed a strong decline in REM sleep with age [32•]. In addition, also as in mammals, the latency from sleep onset to REM sleep increased with age. Although additional species need to be studied, the results from owls suggest that a high amount of REM sleep early in life is another fundamental feature of sleep shared by mammals and birds. As proposed for mammals [52, 53], REM sleep may therefore also play a role in brain development in birds.

\section{Convergent and Divergent Evolution of NREM and REM Sleep}

\section{Convergent Evolution}

The similarities between mammalian and avian sleep either reflect inheritance from a common ancestor with similar states or the result of convergent evolution. Early studies of reptiles and amphibians aimed to answer this question. (Note: Although birds are members of Dinosauria, and therefore Reptilia, to avoid confusion, we will continue to (incorrectly) use the term reptile to refer to all members of this group except birds). If these states were inherited from the most recent common ancestor of mammals and birds (i.e., the stem amniote), then all of its descendants, including all living reptiles, should show similar states. In contrast, the absence of these states in reptiles and amphibians would indicate their convergent evolution in the ancestors of mammals and those of birds. Although discrepancies exist in the literature, most studies on reptiles report a single sleep state characterized by low-amplitude EEG activity, intermittently punctuated by high-voltage spikes (HVS) [6, 54]. Pharmacological studies suggest that HVS are homologous to similar spikes (i.e., sharp waves) occurring in the mammalian hippocampus during NREM sleep. If correct, then one would expect similar spikes to occur in the avian hippocampus during NREM sleep; however, such activity has not been reported. Instead, HVS might represent short and infrequently occurring on-states homologous to the longer, more frequently occurring onstates found in mammals and birds. Although additional studies are needed to resolve this question, both scenarios suggest that sleep in reptiles and amphibians reflects a "rudimentary" form of NREM sleep present in the shared ancestor of amphibians, mammals, reptiles, and birds, that was retained in living amphibians and reptiles, and elaborated upon independently in mammals and birds.

REM sleep also appears to have evolved independently in mammals and birds. Although reports of REM sleep in reptiles have been made, based largely on the presence of "REMlike" twitching [54, 55], most studies fail to report similar movements, nor the presence of REM sleep [56]. Also, studies in "primitive" mammals and birds suggest that REM sleep, as we typically think of it, with EEG activation evolved later than NREM sleep in each group. Monotreme mammals (the egglaying echidnas and platypus) and Palaeognathic birds (ostriches, emus, etc.) are descendants of early branches of the mammalian and avian tree, respectively. Although all living animals continue to evolve [7], monotremes and Palaeognathic birds retain some primitive traits, and therefore might exhibit primitive sleep traits as well. Interestingly, the platypus (Ornithorhynchus anatinus) [57] and ostrich (Struthio camelus) [34] show similar mixed sleep states characterized by features of REM sleep controlled by the brainstem (reduced muscle tone and rapid eye movements) occurring concurrently with slow waves typical of NREM sleep in the forebrain. And, in the echidna (Tachyglossus aculeatus), neocortical slow waves occur concurrently with irregular brainstem neuronal activity similar to that observed during REM sleep in placental mammals [58]. In ostriches [34], and perhaps monotremes [59], periods of EEG activation 
typical of traditional REM sleep also occur during periods of brainstem REM sleep, but these are short when compared to the periods with EEG slow waves. Although more species need to be examined, these studies tentatively suggest that the brainstem-mediated aspects of REM sleep evolved first, and later became associated specifically with periods of forebrain activation in both mammalian and avian lineages. If correct, the evolution of this integrated brainstem/forebrain REM sleep state may be linked to new sleep functions found only in mammals and birds.

\section{Divergent Evolution}

Given that NREM and REM sleep evolved convergently in mammals and birds, it is perhaps not surprising that salient differences also exist between sleep in the two groups. Until recently, these differences remained unexplained and their potential functional implications largely ignored. A framework for interpreting these differences and their implications for understanding mammalian sleep only became apparent when the authors shifted away from model-based and toward comparative-based thinking [9॰].

Several of the brain rhythms observed during sleep in mammals have not been found in studies of birds using recording methods that readily detect such phenomena in mammals [9•]. Although birds exhibit homeostatically regulated slow waves during NREM sleep, mammalian-like thalamocortical spindles have not been described in EEG recordings from a variety of species $[9 \bullet, 24]$. In addition to spindles, the sharp-wave/ripple complexes that occur in the mammalian hippocampus during NREM sleep (and feeding, grooming, and brief pauses in activity) have not been reported in the avian hippocampus during any state. Perhaps, consistent with this difference, the hippocampal theta rhythm observed during active exploration and REM sleep in mammals also seems to be missing in birds [9॰]. Collectively, this suggests that fundamental differences exist between how mammals and birds process hippocampal information during sleep and wakefulness.

In mammals, the neocortical slow oscillation, thalamocortical spindles, and hippocampal sharp-wave/ripple complexes, acting in concert, are thought to transfer information temporarily stored in the hippocampus to the neocortex for long-term storage and integration with preexisting information [60]. The neocortical slow oscillation is thought to orchestrate this process by timing sharp-wave/ripple complexes and thalamocortical spindles, such that they co-occur during the on-state of the oscillation [18, 61]. During sharpwave/ripple complexes neuronal activity patterns that occurred during prior wakefulness are reactivated ("replayed") in a coordinated manner in the hippocampus and neocortex [62]. Thalamocortical spindles occurring during the on-state are thought to provide conditions conducive to the strengthening of the neocortical representation of the memory being reactivated [61]. In this manner, the slow oscillation is thought to orchestrate the transfer of memories. Although several lines of evidence are consistent with this model [60], some aspects have been challenged [63]. However, a comprehensive discussion of the strengths and weaknesses of this model is beyond the scope of this review; our aim here is to simply evaluate it from a comparative perspective.

The differences between avian and mammalian sleeprelated electrophysiology all relate to the systems-level processing of hippocampal information proposed in mammals. Interestingly, the electrophysiological differences might make some sense when viewed in conjunction with the neuroanatomical differences between mammals and birds. As in mammals, the avian hippocampus is involved in processing spatial information; however, the scope of information reaching the avian hippocampus seems far more limited [9॰]. In mammals, the hippocampus receives multi-modal input funneling in from much of the neocortex via high-order association regions, and is thought to use this information to rapidly form associations that can be accessed to recall episodic memories of past experiences in humans, or "episodic-like" (what, where, and when) memories in non-human animals [64]. In contrast, although birds exhibit episodic-like memory [65], the avian hippocampus only receives visual and olfactory information; high-order, multi-modal association regions in the avian brain implicated in orchestrating complex cognitive tasks, including those performed by the mammalian prefrontal cortex (PFC) [66], do not project to the avian hippocampus, or regions providing it with direct input $[9 \bullet, 67]$. Notably, in contrast to the mammalian PFC which receives direct input from the hippocampus and is thought to influence the flow of information in and out of the hippocampus via its projections to regions providing it with direct input, the nidopallium caudolaterale - the avian analogue of the PFC - does not receive direct input from the hippocampus and does not project to regions providing it with input $[9 \bullet, 67]$. This suggests that the information reaching the hippocampus, and the nature of memories formed therein, are fundamentally different between mammals and birds [68]. Finally, in contrast to mammals where the recall of information initially dependent upon the hippocampus may rely less on this structure and more on the neocortex over time, to our knowledge, there is no evidence indicating a similar systems-level processing of hippocampal information in birds [9•]. Albeit often based on the absence of evidence for various phenomena, collectively, these differences seemingly lend comparative support to the systems-level model of sleep's role in processing hippocampal memories in mammals; birds have no need for the rhythms implicated in this process because they do not form the same types of hippocampal memories, and do not seem to transfer the types of memories that the avian hippocampus does process. 
Nonetheless, this comparison also suggests that slow waves (i.e., slow oscillations) likely perform functions unrelated to the systems-level processing of hippocampal memories in both birds and mammals. This could include the systems-level processing of other types of information between other brain regions, as suggested for imprinting memories $[69,70]$. The traveling aspect of slow waves found in mammals and recently described in birds could, in theory, support such processes [8•]. Other types of memory processing linked to sleep in birds [71, 72•] could also involve processing at the systems level, although this remains unknown. In contrast to systems-level hypotheses, the local use-dependent homeostatic regulation of SWA in mammals and birds [73], suggests that slowwaves may be involved in, or at least reflect, processes occurring locally in the brain. Restorative theories suggest that cellular resources depleted, or at risk of becoming depleted, are restored and conserved during the off-state of the slow oscillation [16•]. Similarly, sleep may play a role in cleansing the brain of potentially damaging byproducts resulting from neuronal activity occurring during wakefulness [74]. Such theories alone, however, do not explain the presence of onstates, which due to their awake-like properties, presumably use cellular resources and create damaging byproducts, rather than restore and remove them, respectively. This is especially true for mammals and birds which, during the course of evolution, independently increased the time spent in NREM sleep-related on-states, and independently evolved REM sleep, a persistent wake-like on-state. From a strictly restorative perspective, this is even more paradoxical when one considers the fact that due to their high metabolic rate and behavioral complexity, mammals and birds might be expected to have an even greater need for cellular restoration and energy conservation than ectothermic animals. Clearly, on-states and associated rhythms must serve an important function $[16 \bullet, 75,76]$ presumably needed to a greater extent in mammals and birds when compared to ectotherms. Functional theories for NREM sleep on-states primarily focus on local changes in synaptic strength; however, it is debated whether this reflects primarily the strengthening of connections (perhaps via the reactivation of memories) [60, 71, 76, 77], a weakening of connections (synaptic down-scaling) [13], or a combination of the two [60]. Finally, up to this point, we have focused on on- and off-states as performing separate functions. However, it has also been suggested that the entire slow oscillation per se is needed to process information, either through providing a temporal frame for coordinating activity across brain regions during the systems-level processing of information [61], or creating local conditions conducive to long-term potentiation [76]. Although the exact function of the slow oscillation remains debated, the independent elaboration of sleep-related on-states (both in NREM and REM sleep) in mammals and birds provides a largely untapped opportunity to gain further insight into the functions of these paradoxical wake-like states.

\section{Adaptive Sleeplessness?}

Finally, in addition to the convergent and divergent aspects of avian sleep, birds are also interesting from a comparative perspective because several species engage in non-stop behaviors that seemingly leave little time for sleep [78]. For example, several species engage in flights that last days, weeks, or longer as recently reported in the Alpine swift (Tachymarptis melba) using accelerometry [79]. Based largely on the wealth of evidence underscoring the need for sleep, it has been assumed that such birds must sleep in flight. As in continuously active dolphins [80, 81], some birds might maintain performance during long flights by sleeping unihemispherically [82]; however, the EEG recordings needed to detect sleep in flight have not been performed [78] Moreover, a recent EEG-based study of pectoral sandpipers (Calidris melanotos) raises the possibility that birds engaged in long flights might have evolved a capacity to suspend sleep.

Pectoral sandpipers are a polygynous species that breeds under the constant light of the Arctic summer [11••]. As in all polygynous species [83], the reproductive success of a male depends on successfully competing with other males for territories via displays and physical fights, displaying effectively to choosy females to convince them to mate, remaining vigilant for intruding males and newly arriving females, and acquiring the energy needed to sustain these behaviors. On the other hand, given that a female only lays one clutch of eggs per year and receives no help in raising her young, she can only maximize her evolutionary fitness by choosing the genetically best male to sire her young. Indeed, in evolutionary biology, it is well-established that under such circumstances, selection should favor females that are astute at assessing small differences in the ability of males to perform innate and learned behaviors, differences that presumably reflect variation in the males' genetic quality $[84,85]$. When combined with constant daylight, this creates a situation wherein sexual selection should favor the evolution of males that can curtail sleep while maintaining high neurobehavioral performance.

In an entirely field-based study, using activity monitoring, EEG/EMG recordings, and paternity testing, it was recently shown that during the period when females were fertile, males greatly reduced the time spent sleeping. In the most extreme case, a male was active greater than $95 \%$ of the time for 19 days. Importantly, the males who slept the least were significantly more likely to sire young [11••]. Although performance was not measured directly (at least by the investigators), given that reproductive success integrates performance on a variety of tasks under highly competitive and selective circumstances, males that sired the most young maintained high neurobehavioral performance despite sleeping the least.

How do sleepless males do it? Although the males that slept the least showed the greatest SWA during sleep, suggesting that homeostatic sleep mechanisms were still operating under these 
conditions, calculations of slow-wave energy (i.e., total sleeprelated SWA) indicated that this increase in sleep intensity was far from sufficient to account for the lost sleep and increased time awake, let alone the increase in brain use that might have occurred during wakefulness under these intense circumstances. While it is possible that the males obtained the remaining "missing" sleep by engaging in local sleep (i.e., off-states) while behaviorally awake, as recently shown in rats [42], this also seems unlikely. In contrast to rats wherein local sleep only occurred during immobility and caused reduced performance on a foraging task, the sandpipers were constantly active when not sleeping, and their high reproductive success, which entails a high level of neurobehavioral performance, suggests that they were not similarly compromised.

Why don't all males stay awake? Several factors might account for the male-male variation in sleep duration. First, it is possible that all males have the capacity to do this, but some fall short in their ability to compete with other males for territories and females, due to health, age, etc. As a result, such males may quickly drop out of the competition and resume sleeping to obtain its benefits. Second, male-male variability might reflect ongoing selection for genes that enable males to curtail sleep without experiencing deficits in performance. Indeed, albeit to a lesser extent than in sandpipers, genetic variation in specific genes has been linked to inter-individual variability in resistance to sleep loss in humans [86]. Third, it is also possible that male-male variation in sleep duration reflects different genetically determined reproductive strategies. In this scenario, the long-sleeping phenotype could persist if there are long-term costs resulting from sleep loss, such as reduced lifespan; i.e., males that sleep more may have a lower chance of reproducing within a given year, but may live longer and therefore, over their lifespan, produce similar numbers of offspring to short-sleeping, short-lived males. Ultimately, in contrast to other models of sleepless animals created in the laboratory, which have not undergone the scrutiny of natural selection in the real world, determining the genetic basis of adaptive sleeplessness in pectoral sandpipers in the wild may provide novel insight into the mechanisms and functions of sleep, as well as the ongoing debate over the relationship between sleep duration and longevity in humans [87].

\section{Conclusions}

Birds provide an imperfect model of human sleep and therein lay their value for understanding human sleep. From a comparative perspective, the mosaic of convergently and divergently evolved sleep traits found in mammals and birds provides novel insight into the mechanisms and function of sleep in mammals (Fig. 1). The high amounts of REM sleep early in life, it's persistence into adulthood, and rebounds in REM sleep following sleep deprivation in both mammals and birds, suggest that these are fundamental features of REM sleep. Parsimony would suggest that avian and mammalian REM sleep serve the same fundamental function, a function needed extensively early in life and to a lesser extent during adulthood (e.g., [88]). As for NREM sleep, in mammals, the slow oscillation is thought to orchestrate the transfer of information from the hippocampus to the neocortex. However, the presence of slow oscillations in birds despite the marked differences in the hippocampal memory system, suggests that the slow oscillation likely also plays a more fundamental role in both taxa. The local, use-dependent homeostatic regulation of SWA in mammals and birds suggests a shared fundamental role in cellular restoration and/or synaptic scaling occurring locally in the brain. Finally, comparisons with reptiles suggest that mammals and birds independently increased the amount of sleep time spent in seemingly paradoxical wake-like onstates during NREM and REM sleep. This might be functionally interrelated with the independent evolution of homeothermy, large brains (relative to body size), and complex cognitive abilities in mammals and birds [29]. Further comparisons between sleep in mammals and birds, and across the entire animal kingdom, will likely yield further insight not readily

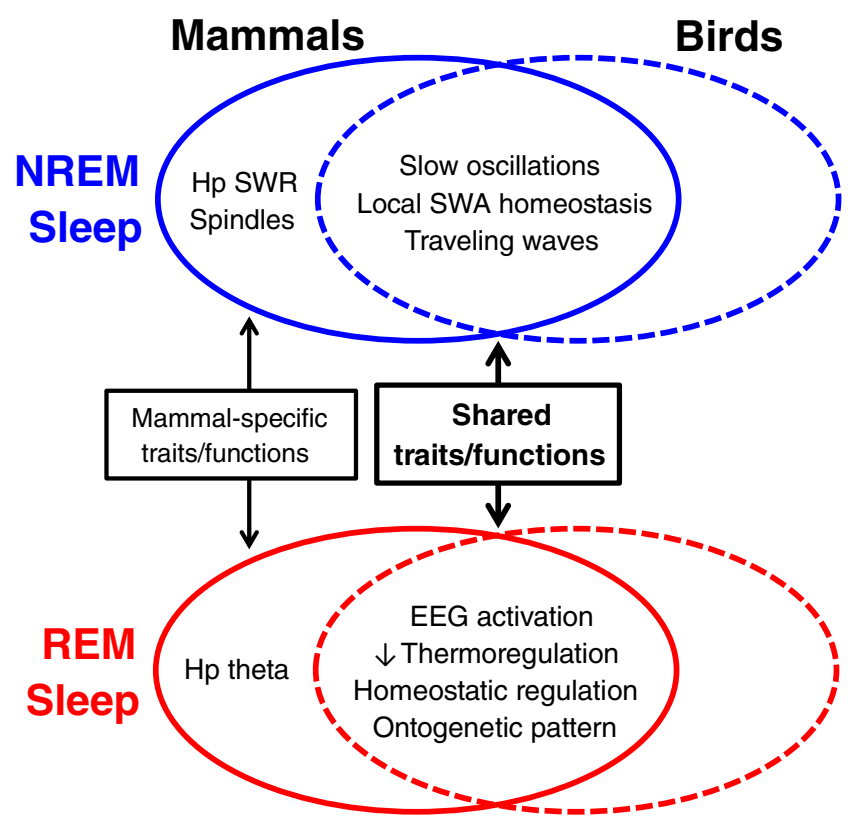

Fig. 1 Venn diagram showing non-rapid eye movement (NREM) and rapid eye movement (REM) sleep traits specific to mammals, and those shared by mammals and birds. Acting in concert with the slow oscillation (a trait shared between mammals and birds), the mammal-specific NREM sleep traits have been implicated in the systems-level processing of hippocampal (Hp) information. The apparent absence of these traits in birds, as well as similar forms of hippocampal processing, suggests that the slow oscillation likely also plays a more fundamental function in avian and mammalian brains. Such functions may be related to the local use-dependent homeostatic regulation of slow-wave activity (SWA), a trait shared by mammals and birds. Similar comparative reasoning can be applied to the mammal-specific and shared aspects of REM sleep. $E E G$ electroencephalogram, SWR sharp-wave/ripples 
obtainable using a traditional model-based approach. A greater recognition by sleep researchers and funding agencies of the heuristic value inherent in exploring both the similarities and the differences between sleep across taxa will likely further our understanding of the evolution and functions of sleep in general. Novel hypotheses generated from this comparative approach, and tested using model-based approaches, will likely translate to an improved understanding of human sleep and waking performance during health and illness.

Acknowledgments This work was supported by the Max Planck Society.

\section{Compliance with Ethics Guidelines}

Conflict of Interest Dr. Rattenborg and Dr. Martinez-Gonzalez declare that they have no conflict of interest.

Human and Animal Rights and Informed Consent This article does not contain any studies with human or animal subjects performed by any of the authors.

\section{References}

Papers of particular interest, published recently, have been highlighted as:

- Of importance

- Of major importance

1. Hendricks JC, Sehgal A. Why a fly? Using drosophila to understand the genetics of circadian rhythms and sleep. Sleep. 2004;27:334-42.

2. Bushey D, Cirelli C. From genetics to structure to function: exploring sleep in Drosophila. Int Rev Neurobiol. 2011;99:213-44.

3. van Alphen B, Yap MH, Kirszenblat L, et al. A dynamic deep sleep stage in Drosophila. J Neurosci. 2013;33:6917-27.

4. Manger PR, Cort J, Ebrahim N, et al. Is 21 st century neuroscience too focused on the rat/mouse model of brain function and dysfunction? Front Neuroanat. 2008;2:5.

5. Striedter GF, Belgard TG, Chen CC, et al. NSF workshop report: discovering general principles of nervous system organization by comparing brain maps across species. J Comp Neurol. 2014;522: $1445-53$.

6. Rattenborg NC. Response to commentary on evolution of slow-wave sleep and palliopallial connectivity in mammals and birds: a hypothesis. Brain Res Bull. 2007;72:187-93.

7. Smulders TV. The relevance of brain evolution for the biomedical sciences. Biol Lett. 2009;5:138-40.

8. • Beckers GJ, van der Meij J, Lesku JA, Rattenborg NC. Plumes of neuronal activity propagate in three dimensions through the nuclear avian brain. BMC Biol. 2014;12:16. Anesthesia-based study demonstrating that, as in mammals, NREM sleep-related neuronal activity propagates as traveling waves through the avian brain despite marked differences in neuronal cytoarchitecture.

9. • Rattenborg NC, Martinez-Gonzalez D, Roth 2nd TC, Pravosudov VV. Hippocampal memory consolidation during sleep: a comparison of mammals and birds. Biol Rev Camb Philos Soc. 2011;86:658-91. A comprehensive review providing a new framework for interpreting differences between avian and mammalian sleep related to the proposed systems-level processing of hippocampal memories.
10. Mukhametov LM, Supin AY, Polyakova IG. Interhemispheric asymmetry of the electroencephalographic sleep patterns in dolphins. Brain Res. 1977;134:581-4.

11. • Lesku JA, Rattenborg NC, Valcu M, et al. Adaptive sleep loss in polygynous pectoral sandpipers. Science. 2012;337:1654-8. Fieldbased study providing direct evidence for adaptive sleep loss in animals performing in a highly competitive real-world situation. These findings demonstrate that reduced neurobehavioral performance is not an evolutionarily inescapable outcome of sleep loss.

12. Krueger JM, Obál F. A neuronal group theory of sleep function. J Sleep Res. 1993;2:63-9.

13. Tononi G, Cirelli C. Sleep and synaptic homeostasis: a hypothesis Brain Res Bull. 2003;62:143-50.

14. Lima SL, Rattenborg NC. A behavioural shutdown can make sleeping safer: a strategic perspective on the function of sleep. Anim Behav. 2007;74:189-97.

15. Van Dongen HP, Belenky G, Krueger JM. A local, bottom-up perspective on sleep deprivation and neurobehavioral performance. Curr Top Med Chem. 2011;11:2414-22.

16. - Vyazovskiy VV, Harris KD. Sleep and the single neuron: the role of global slow oscillations in individual cell rest. Nat Rev Neurosci. 2013;14:443-51. Most recent rendition of a series of functional theories, initially inspired by the discovery of unihemispheric sleep in dolphins, with direct implications for understanding reduced neurobehavioral performance in sleep deprived humans.

17. - Schmidt MH. The energy allocation function of sleep: a unifying theory of sleep, torpor, and continuous wakefulness. Neurosci Biobehav Rev. 2014;47C:122-53. A provocative unifying theory for the function of sleep with biomedical implications inspired, in part, by comparative research on birds.

18. Steriade M. Grouping of brain rhythms in corticothalamic systems. Neurosci. 2006;137:1087-106.

19. Reiner A, Stern EA, Wilson CJ. Physiology and morphology of intratelencephalically projecting corticostriatal-type neurons in pigeons as revealed by intracellular recording and cell filling. Brain Behav Evol. 2001;58:101-14.

20. Nir Y, Staba RJ, Andrillon T, et al. Regional slow waves and spindles in human sleep. Neuron. 2011;70:153-69.

21. Szymczak JT, Kaiser W, Helb HW, Beszczynska B. A study of sleep in the European blackbird. Physiol Behav. 1996;60:1115-20.

22. Rattenborg NC, Mandt BH, Obermeyer WH, et al. Migratory sleeplessness in the white-crowned sparrow (Zonotrichia leucophrys gambelii). PLoS Biol. 2004;2:E212.

23. Low PS, Shank SS, Sejnowski TJ, Margoliash D. Mammalian-like features of sleep structure in zebra finches. Proc Natl Acad Sci U S A. 2008;105:9081-6.

24. Tobler I, Borbély AA. Sleep and EEG spectra in the pigeon (Columba livia) under baseline conditions and after sleep-deprivation. J Comp Physiol A. 1988;163:729-38.

25. Martinez-Gonzalez D, Lesku JA, Rattenborg NC. Increased EEG spectral power density during sleep following short-term sleep deprivation in pigeons (Columba livia): evidence for avian sleep homeostasis. J Sleep Res. 2008;17:140-53.

26. Tobler I, Jaggi K. Sleep and EEG spectra in the Syrian hamster (Mesocricetus auratus) under baseline conditions and following sleep deprivation. J Comp Physiol A. 1987;161:449-59.

27. Jones SG, Vyazovskiy VV, Cirelli C, et al. Homeostatic regulation of sleep in the white-crowned sparrow (Zonotrichia leucophrys gambelii). BMC Neurosci. 2008;9:47.

28. Tobler I. Phylogeny of sleep regulation. In: Kryger MH, Roth T, Dement WC, editors. Principles and practice of sleep medicine. 4th ed. Philadelphia: Elsevier Saunders; 2005. p. 77-90.

29. Rattenborg NC, Martinez-Gonzalez D, Lesku JA. Avian sleep homeostasis: convergent evolution of complex brains, cognition and sleep functions in mammals and birds. Neurosci Biobehav Rev. 2009;33:253-70. 
30. Huber R, Ghilardi MF, Massimini M, et al. Local sleep and learning. Nature. 2004;430:78-81.

31. • Lesku JA, Vyssotski AL, Martinez-Gonzalez D, et al. Local sleep homeostasis in the avian brain: convergence of sleep function in mammals and birds? Proc Biol Sci. 2011;278:2419-28. First experimental evidence for the local, use-dependent regulation of NREM sleep-related EEG SWA in birds.

32. - Scriba MF, Ducrest AL, Henry I, et al. Linking melanism to brain development: expression of a melanism-related gene in barn owl feather follicles covaries with sleep ontogeny. Front Zool. 2013;10: 42. Field-based EEG study providing the first unequivocal evidence for a mammalian-like age-related decline in REM sleep during development in birds.

33. Dewasmes G, Cohen-Adad F, Koubi H, Le Maho Y. Polygraphic and behavioral study of sleep in geese: existence of nuchal atonia during paradoxical sleep. Physiol Behav. 1985;35:67-73.

34. Lesku JA, Meyer LC, Fuller A, et al. Ostriches sleep like platypuses. PLoS One. 2011;6:e23203.

35. Heller HC, Graf R, Rautenberg W. Circadian and arousal state influences on thermoregulation in the pigeon. Am J Physiol. 1983;245:R321-8.

36. Graf R, Heller HC, Sakaguchi S, Krishna S. Influence of spinal and hypothalamic warming on metabolism and sleep in pigeons. Am J Physiol. 1987;252:R661-7.

37. Szymczak JT. Influence of environmental temperature and photoperiod on temporal structure of sleep in corvids. Acta Neurobiol Exp (Wars). 1989;49:359-66.

38. Khalsa SB, Conroy DA, Duffy JF, et al. Sleep- and circadiandependent modulation of REM density. J Sleep Res. 2002;11:53-9.

39. Newman SM, Paletz EM, Rattenborg NC, et al. Sleep deprivation in the pigeon using the disk-over-water method. Physiol Behav. 2008;93:50-8.

40. Newman SM, Paletz EM, Obermeyer WH, Benca RM. Sleep deprivation in pigeons and rats using motion detection. Sleep. 2009;32: 1299-312.

41. Rechtschaffen A, Bergmann BM. Sleep deprivation in the rat: an update of the 1989 paper. Sleep. 2002;25:18-24.

42. Vyazovskiy VV, Olcese U, Hanlon EC, et al. Local sleep in awake rats. Nature. 2011;472:443-7.

43. Roffwarg HP, Muzio JN, Dement WC. Ontogenetic development of the human sleep-dream cycle. Science. 1966;152:604-19.

44. Jouvet-Mounier D, Astic L, Lacote D. Ontogenesis of the states of sleep in rat, cat, and guinea pig during the first postnatal month. Dev Psychobiol. 1970;2:216-39.

45. Frank MG, Heller HC. Development of REM and slow wave sleep in the rat. Am J Physiol. 1997;272:R1792-9.

46. Thurber A, Jha SK, Coleman T, Frank MG. A preliminary study of sleep ontogenesis in the ferret (Mustela putorius furo). Behav Brain Res. 2008;189:41-51.

47. Astic L, Jouvet-Mounier D. Demonstration of paradoxical sleep in utero in guinea pigs. C R Acad Sci. 1969;269:2578-81.

48. Ruckebusch Y. Development of sleep and wakefulness in the foetal lamb. Electroencephalogr Clin Neurophysiol. 1972;32:119-28.

49. Astic L, Sastre JP, Brandon AM. Polygraphic study of vigilance states in the guinea pig fetus. Physiol Behav. 1973;11:647-54.

50. Ibuka N. Ontogenesis of circadian sleep-wakefulness rhythms and developmental changes of sleep in the altricial rat and in the precocial guinea pig. Behav Brain Res. 1984;11:185-96.

51. Szeto HH, Hinman DJ. Prenatal development of sleep-wake patterns in sheep. Sleep. 1985;8:347-55.

52. Shaffery JP, Lopez J, Roffwarg HP. Brain-derived neurotrophic factor (BDNF) reverses the effects of rapid eye movement sleep deprivation (REMSD) on developmentally regulated, long-term potentiation (LTP) in visual cortex slices. Neurosci Lett. 2012;513:84-8.

53. Tiriac A, Uitermarkt BD, Fanning AS, et al. Rapid whisker movements in sleeping newborn rats. Curr Biol. 2012;22:2075-80.
54. Hartse KM. Sleep in insects and nonmammalian vertebrates. In: Kryger MH, Roth T, Dement WC, editors. Principles and practice of sleep medicine. 2nd ed. Philadelphia: WB Saunders Co; 1994. p. 95-104.

55. Ayala-Guerrero F, Mexicano G. Sleep and wakefulness in the green iguanid lizard (Iguana iguana). Comp Biochem Physiol A Mol Integr Physiol. 2008;151:305-12.

56. Eiland MM, Lyamin OI, Siegel JM. State-related discharge of neurons in the brainstem of freely moving box turtles, Terrapene carolina major. Arch Ital Biol. 2001;139:23-36.

57. Siegel JM, Manger PR, Nienhuis R, et al. Sleep in the platypus. Neurosci. 1999;91:391-400.

58. Siegel JM, Manger PR, Nienhuis R, et al. The echidna Tachyglossus aculeatus combines REM and non-REM aspects in a single sleep state: implications for the evolution of sleep. J Neurosci. 1996;16: 3500-6.

59. Nicol SC, Andersen NA, Phillips NH, et al. The echidna manifests typical characteristics of rapid eye movement sleep. Neurosci Lett. 2000;283:49-52.

60. Rasch B, Born J. About sleep's role in memory. Physiol Rev. 2013;93:681-766.

61. Mölle M, Eschenko O, Gais S, et al. The influence of learning on sleep slow oscillations and associated spindles and ripples in humans and rats. Eur J Neurosci. 2009;29:1071-81.

62. Ji D, Wilson MA. Coordinated memory replay in the visual cortex and hippocampus during sleep. Nat Neurosci. 2007;10:100-7.

63. Tononi G, Cirelli C. Sleep and the price of plasticity: from synaptic and cellular homeostasis to memory consolidation and integration. Neuron. 2014;81:12-34.

64. Crystal JD. Episodic-like memory in animals. Behav Brain Res. 2010;215:235-43.

65. Salwiczek LH, Watanabe A, Clayton NS. Ten years of research into avian models of episodic-like memory and its implications for developmental and comparative cognition. Behav Brain Res. 2010;215: 221-34.

66. Kirsch JA, Güntürkün $\mathrm{O}$, Rose J. Insight without cortex: lessons from the avian brain. Conscious Cogn. 2008;17:475-83.

67. Shanahan M, Bingman VP, Shimizu T, et al. Large-scale network organization in the avian forebrain: a connectivity matrix and theoretical analysis. Front Comput Neurosci. 2013;7:89.

68. Rattenborg NC, Martinez-Gonzalez D. A bird-brain view of episodic memory. Behav Brain Res. 2011;222:236-45.

69. Jackson C, McCabe BJ, Nicol AU, et al. Dynamics of a memory trace: effects of sleep on consolidation. Curr Biol. 2008;18:393-400.

70. Beckers GJL, Rattenborg NC. An in depth view of avian sleep. Neurosci Biobehav Rev, in press.

71. Margoliash D, Schmidt MF. Sleep, off-line processing, and vocal learning. Brain Lang. 2010;115:45-58.

72. - Brawn TP, Nusbaum HC, Margoliash D. Sleep consolidation of interfering auditory memories in starlings. Psychol Sci. 2013;24: 439-47. Behavioral study demonstrating a link between memory consolidation and sleep in birds.

73. Rattenborg NC, Lima SL, Lesku JA. Sleep locally, act globally. Neuroscientist. 2012;18:533-46.

74. Xie L, Kang H, Xu Q, et al. Sleep drives metabolite clearance from the adult brain. Science. 2013;342:373-7.

75. Marshall L, Helgadóttir H, Mölle M, Born J. Boosting slow oscillations during sleep potentiates memory. Nature. 2006;444:610-3.

76. Chauvette S, Seigneur J, Timofeev I. Sleep oscillations in the thalamocortical system induce long-term neuronal plasticity. Neuron. 2012;75:1105-13.

77. Gulati T, Ramanathan DS, Wong CC, Ganguly K. Reactivation of emergent task-related ensembles during slow-wave sleep after neuroprosthetic learning. Nat Neurosci. 2014;17:1107-13.

78. Rattenborg NC. Do birds sleep in flight? Naturwissenschaften. 2006;93:413-25. 
79. Liechti F, Witvliet W, Weber R, Bächler E. First evidence of a 200day non-stop flight in a bird. Nat Commun. 2013;4:2554.

80. Lyamin O, Pryaslova J, Kosenko P, Siegel J. Behavioral aspects of sleep in bottlenose dolphin mothers and their calves. Physiol Behav. 2007;92:725-33.

81. Branstetter BK, Finneran JJ, Fletcher EA, et al. Dolphins can maintain vigilant behavior through echolocation for 15 days without interruption or cognitive impairment. PLoS One. 2012;7:e47478.

82. Rattenborg NC, Lima SL, Amlaner CJ. Half-awake to the risk of predation. Nature. 1999;397:397-8.

83. Andersson M. Sexual selection. Princeton, NJ: Princeton University Press; 1994.
84. Byers J, Hebets E, Podos J. Female mate choice based upon male motor performance. Anim Behav. 2010;79:771-8.

85. Barske J, Schlinger BA, Wikelski M, Fusani L. Female choice for male motor skills. Proc Biol Sci. 2011;278:3523-8.

86. Bachmann V, Klaus F, Bodenmann S, et al. Functional ADA polymorphism increases sleep depth and reduces vigilant attention in humans. Cereb Cortex. 2012;22:962-70.

87. Kripke DF, Langer RD, Elliott JA, et al. Mortality related to actigraphic long and short sleep. Sleep Med. 2011;12:28-33.

88. Bellesi M, Pfister-Genskow M, Maret S, et al. Effects of sleep and wake on oligodendrocytes and their precursors. J Neurosci. 2013;33: $14288-300$ 